\title{
Immune activation alters cellular and humoral responses to yellow fever 17D vaccine
}

Enoch Muyanja, ${ }^{1}$ Aloysius Ssemaganda, ${ }^{1}$ Pearline Ngauv, ${ }^{2}$ Rafael Cubas, ${ }^{2}$ Helene Perrin, ${ }^{2}$ Divya Srinivasan, ${ }^{2}$ Glenda Canderan, ${ }^{2}$ Benton Lawson, ${ }^{3}$ Jakub Kopycinski, ${ }^{4}$ Amanda S. Graham, ${ }^{5}$ Dawne K. Rowe, ${ }^{5}$ Michaela J. Smith, ${ }^{5}$ Sharon Isern, ${ }^{5}$ Scott Michael, ${ }^{5}$ Guido Silvestri, ${ }^{3}$ Thomas H. Vanderford, ${ }^{3}$ Erika Castro, ${ }^{6}$ Giuseppe Pantaleo, ${ }^{6,7}$ Joel Singer, ${ }^{8}$ Jill Gillmour, ${ }^{4}$ Noah Kiwanuka, ${ }^{1,9}$ Annet Nanvubya, ${ }^{1}$ Claudia Schmidt, ${ }^{9}$ Josephine Birungi, ${ }^{1}$ Josephine Cox, ${ }^{4}$ Elias K. Haddad,, ${ }^{2}$ Pontiano Kaleebu,, ${ }^{1,10}$ Patricia Fast, ${ }^{11}$ Rafick-Pierre Sekaly, ${ }^{2}$ and Lydie Trautmann ${ }^{2}$

1 Uganda Virus Research Institute-International AIDS Vaccine Initiative (UVRI-IAVI) HIV Vaccine Program, Uganda Virus Research Institute (UVRI),

Entebbe, Uganda. ${ }^{2}$ Vaccine and Gene Therapy Institute of Florida, Port Saint Lucie, Florida, USA. ${ }^{3}$ Center for AIDS Research Virology Core,

Emory Vaccine Center, Atlanta, Georgia, USA. ${ }^{4}$ International AIDS Vaccine Initiative Human Immunology Laboratory, London, United Kingdom.

5Department of Biological Sciences, Florida Gulf Coast University, Fort Myers, Florida, USA. ${ }^{6}$ Division of Immunology and Allergy, Lausanne, Switzerland.

${ }^{7}$ Swiss Vaccine Research Institute, Lausanne University Hospital, University of Lausanne, Lausanne, Switzerland.

${ }^{8}$ Canadian Institutes of Health Research (CIHR) Canadian HIV Trials Network, Vancouver, British Columbia, Canada.

9Makerere University School of Public Health, Entebbe, Uganda. ${ }^{10 M R C / U V R I ~ U g a n d a ~ R e s e a r c h ~ U n i t ~ o n ~ A I D S, ~ E n t e b b e, ~ U g a n d a . ~}$ ${ }^{11}$ International AIDS Vaccine Initiative, New York, New York, USA.

Background. Defining the parameters that modulate vaccine responses in African populations will be imperative to design effective vaccines for protection against HIV, malaria, tuberculosis, and dengue virus infections. This study aimed to evaluate the contribution of the patient-specific immune microenvironment to the response to the licensed yellow fever vaccine 17D (YF-17D) in an African cohort.

Methods. We compared responses to YF-17D in 50 volunteers in Entebbe, Uganda, and 50 volunteers in Lausanne, Switzerland. We measured the $\mathrm{CD8}^{+} \mathrm{T}$ cell and $\mathrm{B}$ cell responses induced by YF-17D and correlated them with immune parameters analyzed by flow cytometry prior to vaccination.

Results. We showed that YF-17D-induced $\mathrm{CD8}^{+} \mathrm{T}$ cell and $\mathrm{B}$ cell responses were substantially lower in immunized individuals from Entebbe compared with immunized individuals from Lausanne. The impaired vaccine response in the Entebbe cohort associated with reduced YF-17D replication. Prior to vaccination, we observed higher frequencies of exhausted and activated NK cells, differentiated $T$ and $B$ cell subsets and proinflammatory monocytes, suggesting an activated immune microenvironment in the Entebbe volunteers. Interestingly, activation of $\mathrm{CD8}^{+} \mathrm{T}$ cells and $\mathrm{B}$ cells as well as proinflammatory monocytes at baseline negatively correlated with YF-17D-neutralizing antibody titers after vaccination. Additionally, memory $T$ and $B$ cell responses in preimmunized volunteers exhibited reduced persistence in the Entebbe cohort but were boosted by a second vaccination.

Conclusion. Together, these results demonstrate that an activated immune microenvironment prior to vaccination impedes efficacy of the YF-17D vaccine in an African cohort and suggest that vaccine regimens may need to be boosted in African populations to achieve efficient immunity.

Trial registration. Registration is not required for observational studies.

Funding. This study was funded by Canada's Global Health Research Initiative, Defense Threat Reduction Agency, National Institute of Allergy and Infectious Diseases, Bill \& Melinda Gates Foundation, and United States Agency for International Development.

\section{Introduction}

The yellow fever vaccine 17D (YF-17D) induces an efficient immune protection against infection. The protective effect of the vaccine is thought to occur within 10 days after vaccination in $95 \%$ of vaccinees and may persist for up to 30 to 35 years following vaccination (1-4). A booster vaccination is recommended by the US Centers for Disease Control and Prevention after 10 years (5, 6); however, the World Health Organization recommends a single vaccination (7). This vaccine has been used recently to determine a gene expression signature after vaccination that highlights the fea-

Conflict of interest: The authors have declared that no conflict of interest exists. Citation for this article: J Clin Invest. 2014;124(7):3147-3158. doi:10.1172/JCI75429. tures of protective immune responses in North American adults (8, 9). This immune response involves the coordinated induction of several effector arms of innate immunity as well as adaptive immunity $(10,11)$. Studies have suggested that the ability of YF-17D to infect dendritic cells and activate multiple Toll-like receptors may be essential for generating this potent immune response $(8,12-$ 14). The YF-17D vaccine provides a model to study the immune response to vaccination in human populations, including both cellular and humoral responses, and to dissect the mechanisms of effective vaccine responses $(10,15-18)$. Defining vaccine efficacy in African populations is of paramount importance, as these populations are targeted by most of the currently tested vaccines for HIV, tuberculosis, malaria, and dengue virus (DENV) (19). Of note, 

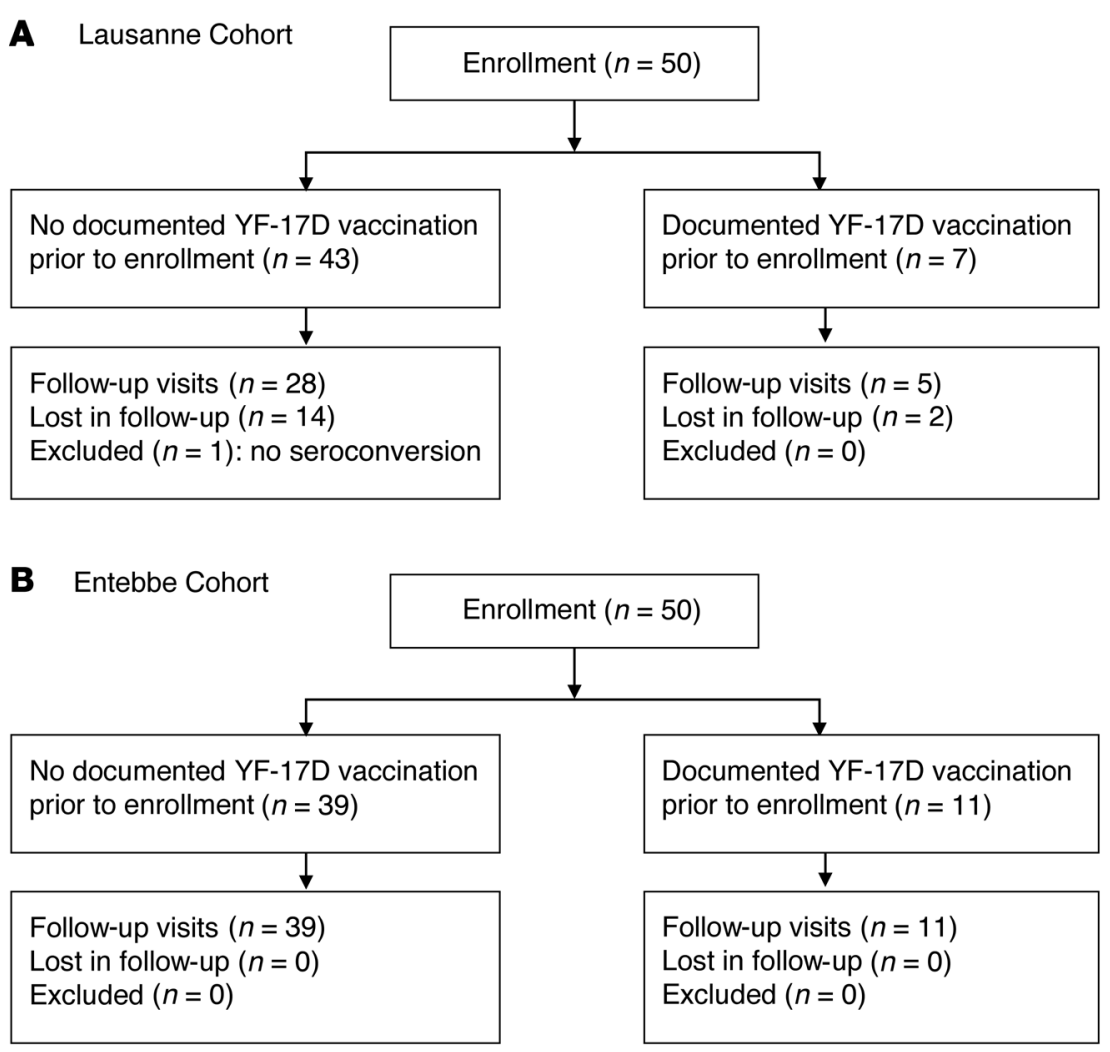

Figure 1

CONSORT diagram for (A) Lausanne and (B) Entebbe cohorts.

that in those from Malawi (26). The authors suggested that differential sensitization due to exposure to environmental mycobacteria between the 2 populations was responsible for the difference in magnitude of BCG responsiveness. BCG vaccination was also shown to induce different cytokine profiles between United Kingdom and Malawi (27). It has furthermore been suggested that there is a reduced immune response to HIV vaccination in African populations when compared with a North American population (B003/HVTN091 study) (28). In the present study, we compared the responses to YF-17D vaccination in 2 cohorts from regions in which yellow fever virus (YFV) was not endemic, Lausanne, Switzerland, and Entebbe, Uganda. By studying the immune responses to YF-17D in a European and an African population, we sought to further understand the impact of the immune microenvironment on vaccine efficacy in 2 different populations.

\section{Results}

Study population. In this study, volunteers were enrolled and vaccinated with the YF-17D vaccine in 2 different cohorts at 2 sites: Entebbe, Uganda, and Lausanne, Switzerland. In Entebbe, 50 participants were assigned to 2 groups (Figure 1 and Table 1): the first vaccination group consisted of 39 volunteers who received the YF-17D vaccine for the first time

several vaccine candidates use the YF-17D backbone as a vector for presentation of antigens from DENV, Japanese encephalitis virus, West Nile virus, and HIV (20-22).

Factors that could affect vaccine responses include genetic background, gender, age, and environmental differences. In a large study comparing the response to YF-17D in healthy adults from different origins, men of mixed European descent showed higher antibody levels when compared with females, individuals of African descent, or Hispanics (23), but this study did not observe age-related differences in vaccine responses. A large clinical trial in North America and the United Kingdom compared YF-17D immunogenicity between adults and elderly individuals and did not report differences in the generation of neutralizing antibodies (NAbs) or differences in other quantitative responses between the two groups (24). However, another study analyzed the YF-17D response in elderly and younger subjects and showed that elderly individuals had a delayed antibody response and higher viremia, but the study did not analyze the persistence of NAb titers (25). These previous studies reported discrete and discordant effects of gender and age on the generation of antibodies against YF-17D, but no studies have investigated the quantitative and qualitative responses to YF-17D in African populations. Some reports have suggested that immune responses to vaccination are weaker in subjects from African populations as compared with that in other groups. Black et al. showed, by comparing two randomized controlled studies in the United Kingdom and Malawi, that Bacillus Calmette-Guerin (BCG) vaccination induced a higher response in individuals from the United Kingdom when compared with at the study; the boost vaccination group included 11 volunteers who had received the YF-17D vaccine at least 6 years prior to enrollment and who received a boost in the study. The volunteers were in good health. Volunteers were sampled at baseline on day 0 and followed for 3 months after enrollment, with visits occurring on days $3,7,10,14,28$, and 84 (Figure $2 \mathrm{~A}$ ). Naive vaccinees reported more reactogenicity compared with those who were boosted (Supplemental Table 1; supplemental material available online with this article; doi:10.1172/JCI75429DS1). All adverse events reported during the course of the study resolved without sequelae (Supplemental Table 2). In Lausanne, 50 participants

\section{Table 1}

Demographics of the 2 cohorts

\begin{tabular}{lccccc}
\hline & \multicolumn{2}{c}{ First vaccination } & \multicolumn{2}{c}{ Boost vaccination } \\
& & Lausanne & Entebbe & Lausanne & Entebbe \\
\multirow{2}{*}{ No. of subjects } & 43 & 39 & 7 & 11 \\
\multirow{2}{*}{ Age } & Mean & 28 & 27 & 39 & 36 \\
& SD & 8 & 6 & 8 & 7 \\
\multirow{2}{*}{ Gender } & Male & 12 & 23 & 4 & 9 \\
& Female & 31 & 16 & 3 & 2 \\
\hline
\end{tabular}

Number of subjects and age and gender of the enrolled volunteers from Lausanne and Entebbe in 2 groups. The first vaccination group received YF-17D vaccine for the first time in the study, and the boost vaccination group had received the YF-17D vaccine prior to the study. 
A

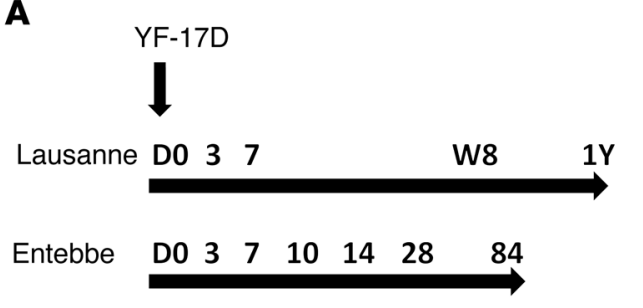

B $\quad \mathrm{v}$

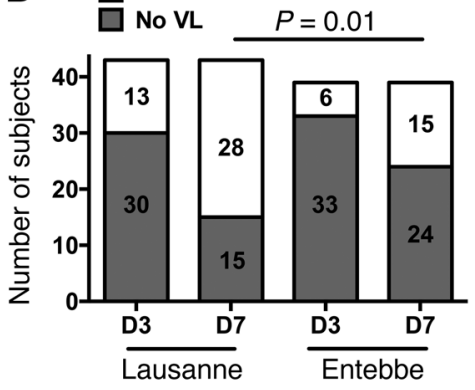

C

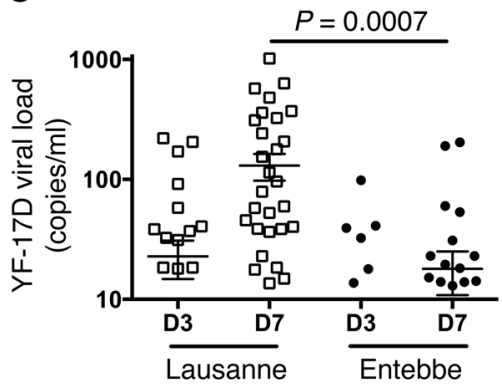

Figure 2

Differences in viremia after YF-17D vaccination in Lausanne and Entebbe. (A) Vaccination schedule in the 2 cohorts and longitudinal sample collection time points used in this study. (B) Number of naive and boosted volunteers with or without detectable viremia in the plasma by quantitative RT-PCR 3 and 7 days after YF-17D vaccination. Numbers of subjects in each group are indicated within the bar graphs. VL, viral load. (C) YF-17D viral load measured in the plasma of naive vaccinees by quantitative RT-PCR 3 and 7 days after vaccination in Lausanne (white squares) and Entebbe (black dots).

were vaccinated and grouped: 43 of the participants were YF-17D naive and 7 were volunteers who had received the YF-17D vaccine at least 10 years prior to enrollment (Figure 1 and Table 1 ). Three individuals experienced adverse events within the vaccine naive group (Supplemental Table 3). Volunteer samples were taken at baseline on day 0 , and volunteers were followed for 1 year after enrollment, with visits occurring at days 3 and 7, week 8, and 1 year (Figure 2A). At each time point in both cohorts, sera, plasma, and PBMCs were collected and processed following standardized standard operating procedures in both sites with good clinical laboratory practice compliance. Samples were frozen on site and shipped between sites for analysis at the required temperatures ( $\mathrm{LN}_{2}$ for PBMCs and $-80^{\circ} \mathrm{C}$ for plasma and sera samples). To limit batch effects, samples from Lausanne and Entebbe were analyzed simultaneously throughout the study. Demographics showed no differences in age between the 2 cohorts, but there was a higher proportion of women in the Lausanne cohort $(P=0.003)$ (Table 1$)$. Gender was therefore tested as a variable in the study, and adjusting for gender made no difference in the analyses.

We measured viremia 3 and 7 days after YF-17D vaccination in the plasma of vaccinees from both cohorts. YF-17D viral load was detected in a higher number of naive vaccinees in Lausanne compared with that in those in Entebbe at day $7(P=0.01)$ (Figure 2B). Peak viral load was detected at day 7 , and almost all individuals exhibited undetectable viral load at day 10 in Entebbe (data not shown). YF-17D vaccination induced significantly higher viral loads in the Lausanne cohort compared with the Entebbe cohort 7 days after vaccination (means of 130 and 18 copies per $\mathrm{ml}$, respectively; $P=0.0007$ ) (Figure $2 \mathrm{C}$ ). These results demonstrate that the YF-17D replication was lower in individuals from Entebbe compared with that in those from Lausanne. Of note, we did not observe a significant association between reactogenicity and viral load in the Entebbe cohort (Supplemental Figure 1). To exclude the possibility that the lower viremia observed in the Entebbe cohort was due to crossNAbs against another flavivirus, we analyzed the neutralization activity against DENV, as it is the most common mosquito-transmitted viral human infection in the world (29). The sera of volunteers in Uganda did not show any neutralization activity against DENV-2 (Supplemental Figure 2, A and B). As secondary or more DENV infections tend to produce broadly neutralizing responses, these data suggest that none of the participants had multiple DENV exposures. Furthermore, NAbs against YF-17D were not detected at baseline in the vaccine-naive recipients, confirming that the lower response in Entebbe was not due to a preexisting immunity.

Heightened activation of the innate compartment in Entebbe vaccinees. As we observed differences in viral replication between the 2 groups of vaccinees, we analyzed the distribution of innate immune subsets that could affect viral replication, such as NK cells and monocytes, at baseline and after vaccination by flow cytometry (Supplemental Figure 3). We did not observe differences in the frequency of $\mathrm{CD} 56^{+} \mathrm{NKp} 46^{+} \mathrm{NK}$ cells within the lymphocytes between the Lausanne and Entebbe cohorts (data not shown). However, significantly higher frequencies of CD $16^{+}$HLA-DR ${ }^{+}$NK cells, considered exhausted NK cells, were detected at day 0, 3, and 7 in the Entebbe cohort when compared with the Lausanne cohort (Figure $3 \mathrm{~A})$. In the Entebbe cohort, NK cells proliferated in response to vaccination, as demonstrated by the increase in the frequency of $\mathrm{Ki}^{+}$ NK cells at day 7 compared with day $0(P=0.04$, Supplemental Figure 4A). This increase in proliferating NK cells was not observed in the Lausanne cohort. We further observed significantly higher frequencies of recently activated CD16- NK cells secreting IFN- $\gamma$ after restimulation ex vivo at all 3 time points in the Entebbe cohort when compared with the Lausanne cohort (Figure 3B). These data were supported by the observation that IFN- $\gamma$ spontaneously secreted by PBMCs taken day 0,3 , and 7 after vaccination was significantly higher in the Entebbe cohort when compared with that in the Lausanne cohort (Supplemental Figure 4B). Significantly higher frequencies of proinflammatory monocytes expressing CD14 and CD16 (30) were also observed at baseline in the Entebbe cohort when compared with those in the Lausanne cohort (means: 11 in the Lausanne cohort and 17 in the Entebbe cohort; $P=0.002$ ) (Figure 3C). Of note, these monocytes expressing CD16 also expressed higher levels of PD-L1 and HLA-DR, corroborating their higher activation state (Supplemental Figure 4C). Overall, these results show a persistent proinflammatory innate compartment in the Entebbe cohort. The heightened activation of multiple innate immune subsets may play a role in the decreased viral replication of YF-17D observed in the Entebbe cohort (Figure 2C). 
A

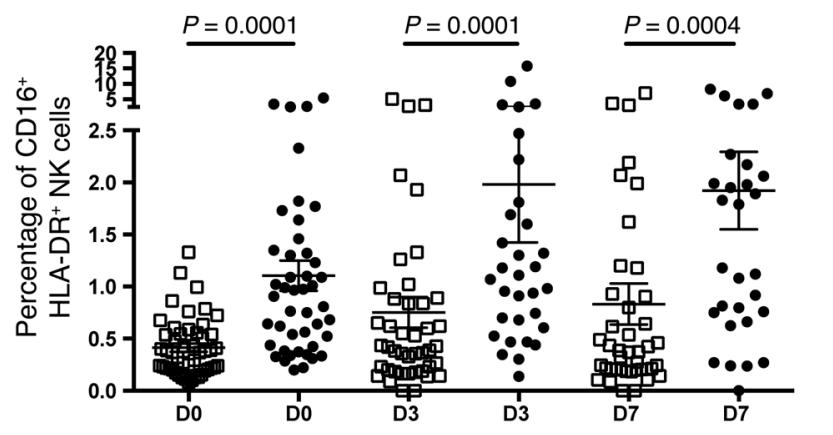

B
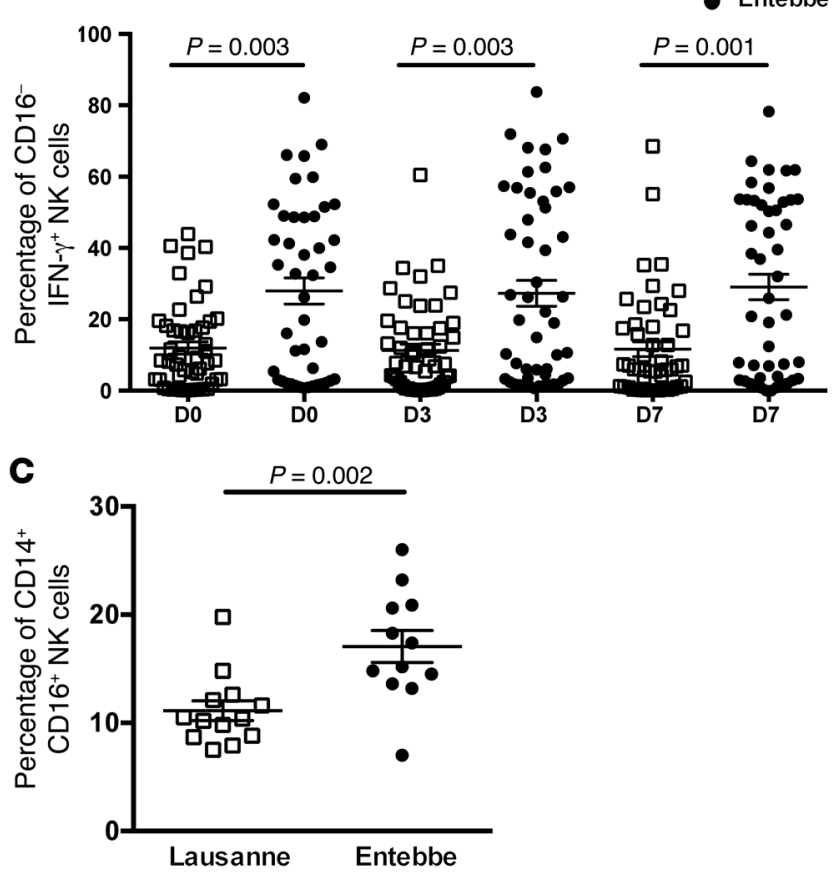

Increased baseline activation of the adaptive compartment in Entebbe vaccinees. We further investigated the adaptive immune response prior to YF-17D vaccination in the 2 cohorts by comparing the cellular distribution of the different $\mathrm{T}$ and $\mathrm{B}$ cell compartments at baseline (Supplemental Figures 5 and 6). In the CD4 T cell compartment, significantly higher frequencies of effector memory CD4 T cells were detected in the Entebbe cohort when compared with the Lausanne cohort (means: 6.1 in the Lausanne cohort and 10.5 in the Entebbe cohort; $P<0.0001$ ) (Figure 4A). The CD8 T cell compartment also showed a distribution toward more differentiated cells, with significantly lower transitional memory CD8 T cells (means: 26.3 in the Lausanne cohort and 18.9 in the Entebbe cohort; $P<0.0001$ ) but significantly higher frequencies of effector memory (means: 3.4 in the Lausanne cohort and 5.1 in the Entebbe cohort; $P=0.003$ ) and terminally differentiated CD8 T cells (means: 5.9 in the Lausanne cohort and 14.8 in the Entebbe cohort; $P<0.0001$ ) in the Entebbe cohort (Figure 4B). No differences were observed in the frequencies of naive CD4 and CD8 T cells between the 2 cohorts (data not shown). We observed higher levels of activation of memory CD4 T cells, as measured by

\section{Figure 3}

Differences in innate cells between Lausanne and Entebbe cohorts. (A) Frequency of exhausted CD16+ ${ }^{+} \mathrm{LA}-\mathrm{DR}{ }^{+} \mathrm{NK}$ cells in CD3- NKp46+ CD56+ NK cells at day 0,3 , and 7 after vaccination in Lausanne and Entebbe cohorts. (B) Frequency of activated CD16- NK cells in CD3$\mathrm{NKp} 46^{+} \mathrm{CD}^{2} 6^{+} \mathrm{NK}$ cells secreting IFN- $\gamma^{+}$after ex vivo restimulation stained by intracellular cytokine staining at day 0,3 , and 7 after vaccination in Lausanne and Entebbe cohorts. (C) Frequency of CD14+ CD16+ inflammatory monocytes at baseline in Lausanne and Entebbe cohorts. White squares represent data points from the Lausanne cohort, and black dots represent data points from the Entebbe cohort.

increased PD-1 (means: 22.1 in the Lausanne cohort and 26.9 in the Entebbe cohort; $P=0.0002$ ) and ICOS expression (means: 2.4 in the Lausanne cohort and 3.2 in the Entebbe cohort; $P=0.003$ ) (Figure 4, C and D). Significantly higher frequencies of activated CD8 T cells expressing CD38 and HLA-DR were also observed in Entebbe vaccinees (means: 0.66 in the Lausanne cohort and 1.48 in the Entebbe cohort; $P<0.0001$ ) (Figure 4E). Of note, no difference was observed in the frequency of CD 4 Tregs between the 2 cohorts at baseline (Figure 4F). This bias toward more differentiated cells and higher levels of activation in both CD4 and CD8 T cells suggests a more activated cellular adaptive compartment at baseline in Entebbe vaccinees when compared with Lausanne vaccinees.

In the B cell compartment, we observed a trend toward higher frequencies of tissue-like memory B cells (defined by CD $19^{+} \mathrm{CD} 27^{-}$ CD21-) as well as activated memory B cells (defined by CD19+ $\left.\mathrm{CD} 27^{+} \mathrm{CD} 21^{-}\right)$in the Entebbe cohort (Figure 5A). CD27- CD21 tissue-like memory B cells show characteristics of exhausted cells, with increased expression of inhibitory receptors and reduced capacity to proliferate $(31,32)$. Additionally, we observed a decrease in the frequency of naive $\mathrm{B}$ cells but no significant differences in the frequency of resting memory B cell subsets between Lausanne and Entebbe cohorts (Figure 5B). Interestingly, we found significantly lower frequencies of $\operatorname{IgM}^{+}$memory B cells (means: 41.4 in the Lausanne cohort and 28.9 in the Entebbe cohort; $P=0.007$ ) and higher frequencies of $\mathrm{IgG}^{+}$memory B cells (means: 28.5 in the Lausanne cohort and 39 in the Entebbe cohort; $P=0.004$ ) in the Entebbe cohort when compared with the Lausanne cohort (Figure 5C). Recent evidence suggests distinct functions between $\operatorname{IgM}^{+}$and $\mathrm{IgG}^{+}$memory B cells: $\mathrm{IgM}^{+}$memory B cells are believed to retain broader antibody specificities, have better survival ability, and, more importantly, can reinitiate germinal center formation during secondary responses $(33,34)$. Thus, the high levels of $\operatorname{IgM}^{+}$memory B cells in the Lausanne cohort could provide more flexibility to the overall humoral immunity. We furthermore analyzed the frequency of plasmablasts, defined by CD38 and CD27 expression prior to YF-17D vaccination, and found a significantly higher frequency of plasmablasts in the Entebbe cohort compared with that in the Lausanne cohort $(P=0.07)$ (Figure 5D). The presence of higher levels of tissue-like memory B cells, activated B cells, $\mathrm{IgG}^{+}$ B cells, and plasmablasts suggests a more activated humoral compartment at baseline in Entebbe vaccinees when compared with Lausanne vaccinees.

Impaired cellular and humoral immune responses to $Y F-17 D$ in Entebbe vaccinees. In order to analyze the impact of an activated immune microenvironment at baseline on the responses induced by the YF-17D vaccine, we measured the cellular and humoral responses following YF-17D vaccination. To study the CD8 T cell response induced by the vaccine, we selected the HLA-A*0201 subjects in 
A

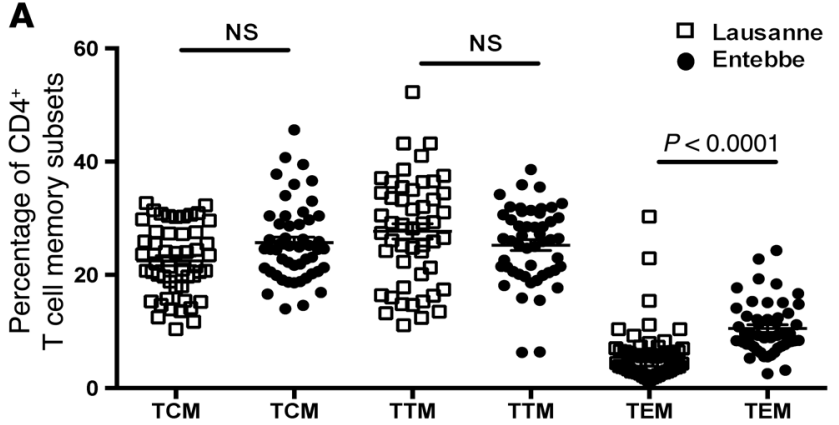

B

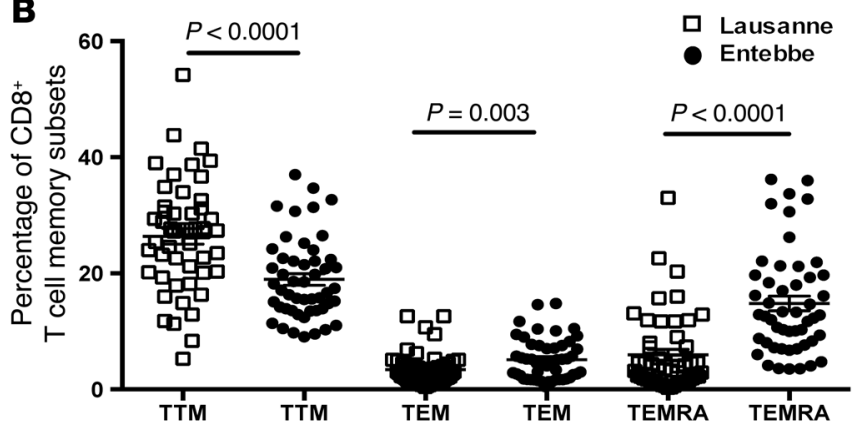

C

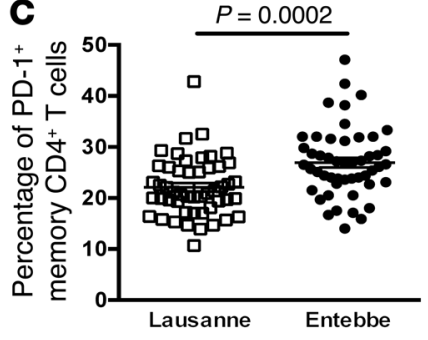

$\mathbf{E}$

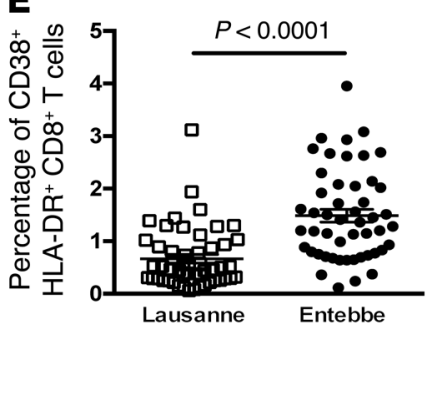

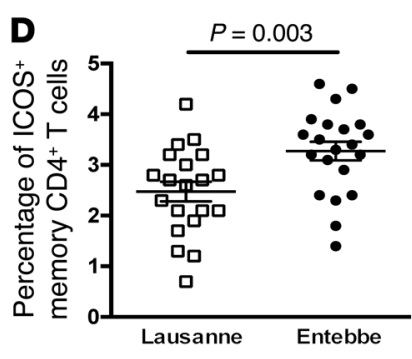

$\mathbf{F}$

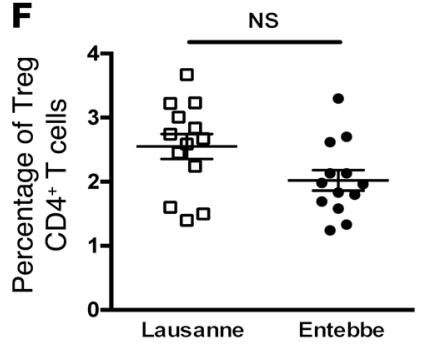

Figure 4

Baseline differences in T cell subsets between Lausanne and Entebbe cohorts. (A) Frequency of CD4 T cell memory subset distribution at baseline in Lausanne and Entebbe cohorts for central memory (TCM), transitional memory (TTM), and effector memory CD4 T cells (TEM), defined by expression of CCR7, CD27, and CD45RA. (B) Frequency of memory CD8 T cell subset distribution at baseline in Lausanne and Entebbe cohorts for transitional memory, effector memory, and terminally differentiated CD8 T cells (TEMRA), defined by expression of CCR7, CD27, and CD45RA. (C) Frequency of activated cells expressing PD-1 within the memory CD4 T cells at baseline in Lausanne and Entebbe cohorts. (D) Frequency of activated cells expressing ICOS within the memory CD4 T cells at baseline in Lausanne and Entebbe cohorts. (E) Frequency of activated cells expressing CD38 and HLA-DR within the memory CD8 T cells at baseline in Lausanne and Entebbe cohorts. (F) Frequency of Treg CD4 T cells, defined as $\mathrm{CD}^{+}{ }^{+} \mathrm{CD} 4{ }^{+} \mathrm{CD} 45 \mathrm{RA}-\mathrm{CD} 25^{+} \mathrm{IL}-7 \mathrm{R}^{-} \mathrm{Foxp}^{+}$, at baseline in Lausanne and Entebbe cohorts. White squares represent data points from the Lausanne cohort, and black dots represent data points from the Entebbe cohort.

both cohorts to circumvent bias due to allele differences (12 in the Lausanne cohort and 7 in the Entebbe cohort) and used the HLA$A * 0201 /$ LV9 tetramer directed against the dominant response to YFV NS4B protein (A2/NS4B) (17, 18, 35). A2/NS4B-specific CD8 $T$ cell responses were measured 1 year after vaccination in the Lausanne cohort and 84 days after vaccination in the Entebbe cohort. Frequencies of A2/NS4B-specific CD8 T cells induced by the vaccine were significantly lower in the Entebbe cohort compared with those in the Lausanne cohort (means: 1.2 in the Lausanne cohort and 0.36 in the Entebbe cohort; $P=0.005$ ) (Figure 6A). To assess the humoral immune response to YF-17D, we measured the YF-17D NAb titers 1 year after vaccination in the 33 donors for which samples were available in Lausanne and 84 days after vaccination in the 50 donors in Entebbe. We observed a strong association between the YF-17D-specific cellular and humoral responses after vaccination, as demonstrated by a significant correlation between A2/NS4B-specific CD8 T cell frequencies and YF-17D NAb titers $(P=0.0003)$ (Figure $6 \mathrm{~B})$. The NAb levels induced by the vaccine were significantly lower in the Entebbe cohort compared with those in the Lausanne cohort (means: -3.5 in the Lausanne cohort and -3.2 in the Entebbe cohort; $P=0.005$ ) (Figure 6C). No significant difference in YF-17D NAb titers was observed in both cohorts in subjects with detectable viremia and those with no detectable viremia at any time point measured, suggesting that the level of virus replication was not the main determinant for the magnitude of the humoral response to YF-17D (Figure 6, D and E). Of note, in the Lausanne cohort, no significant difference was observed in NAb levels between individuals receiving the YF-17D vaccine alone or in combination with other immunizations (Supplemental Figure 7). Adjusting for gender did not show significant differences in NAb titers in the 2 cohorts $(P=0.23)$ or in each cohort separately (Supplemental Table 4), suggesting that this parameter was not a confounding factor in our study. Of note, no association was observed between levels of YF-17D NAb and neutropenia in subjects from Entebbe (Supplemental Table 4).

Association between baseline immune activation and lower response to $Y F-17 D$. As we observed a higher immune activation in the Entebbe cohort at baseline and lower YF-17D response after vaccination, we looked for baseline parameters that could dictate the magnitude of vaccine response among all the immune parameters measured at baseline. We found a strong and significant negative correlation between the frequency of CD $38^{+}$ $\mathrm{CD} 21^{+} \mathrm{CD} 27^{-} \mathrm{B}$ cells, corresponding to either immature transitional B cells or activated naive B cells, and the NAb titers of both cohorts after YF-17D vaccination $(P=0.0004)$ (Figure 7A and Supplemental Figure 8A). A significant association was also found between the frequency of tissue-like memory B cells and lower NAb titers after YF-17D vaccination in both cohorts $(P=0.04)$ (Figure 7B and Supplemental Figure 8B). The cellular adaptive compartment showed a significant negative correla- 

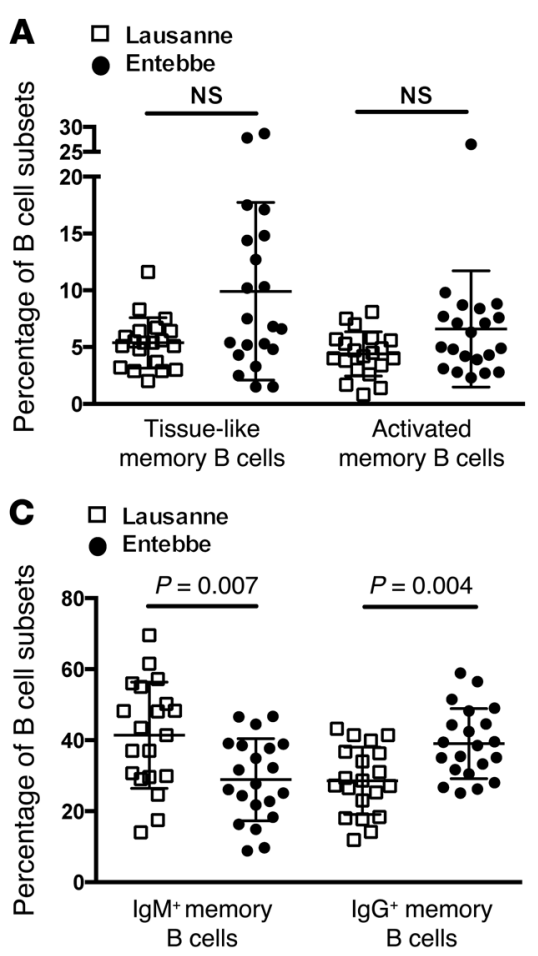

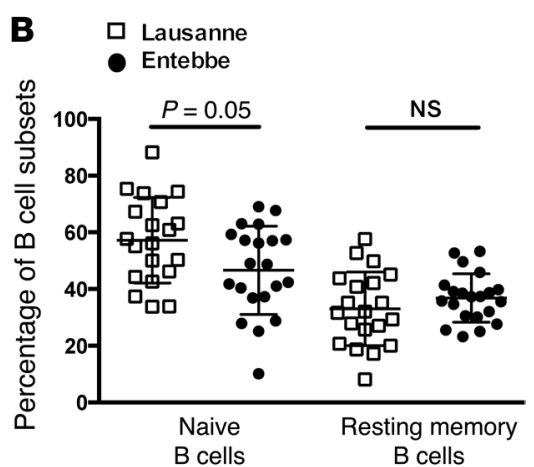

D

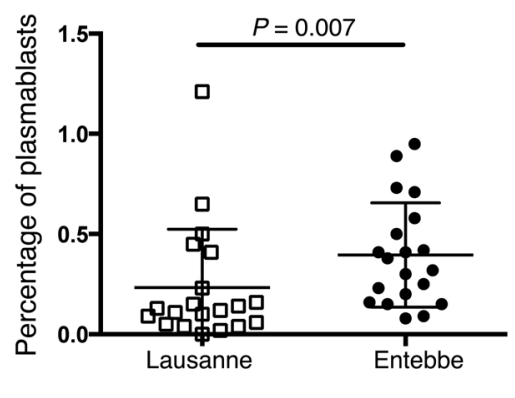

\section{Figure 5}

Baseline differences in $B$ cell subsets between Lausanne and Entebbe cohorts. (A) Frequency of tissue-like memory B cell (CD19+CD27-CD21-) and activated memory $B$ cell (CD19+CD27+CD21-) subsets at baseline in Lausanne and Entebbe cohorts. (B) Frequency of naive (CD19+ CD27-CD21+) and resting memory B cell (CD19+ $\left.\mathrm{CD} 27^{+} \mathrm{CD} 21^{+}\right)$subsets at baseline in Lausanne and Entebbe cohorts. (C) Frequency of $\operatorname{lgM}^{+}$and $\mathrm{IgG}^{+}$memory $\mathrm{B}$ cell subsets at baseline in Lausanne and Entebbe cohorts. (D) Frequency of plasmablasts $\left(\mathrm{CD} 38^{+} \mathrm{CD} 27^{+}\right)$in total $\mathrm{B}$ cells at baseline in Lausanne and Entebbe cohorts. White squares represent data points from the Lausanne cohort, and black dots represent data points from the Entebbe cohort. tion between the expression levels of PD-1 on the memory CD8 $\mathrm{T}$ cell subset and the NAb titers in both cohorts after YF-17D vaccination $(P=0.001)$ (Figure $7 \mathrm{C}$ and Supplemental Figure $8 \mathrm{C})$. Within the innate cell subsets at baseline, the frequency of $\mathrm{CD} 14^{+} \mathrm{CD} 16^{+}$proinflammatory monocytes was associated with lower NAb titers after YF-17D vaccination in both cohorts $(P=0.003)$ (Figure 7D). This association was confirmed by the negative correlation between the NAb titers of the Entebbe cohort and the amount of IL-10 secreted by baseline PBMCs incubated with YF-17D overnight $(P=0.04)$ (Figure 7E). As hematological data were available for the Entebbe cohort, we also found a strong negative correlation between the blood monocyte counts at baseline and the NAb titers after vaccination in the Entebbe cohort $(P=0.0001)$ (Figure 7F). No other hematological data at baseline were associated with NAb titers in the Entebbe cohort (Supplemental Table 5). As full blood data were not available for the Lausanne cohort, we could not perform the same correlation analysis between the absolute count of monocytes and the NAb titers. Altogether, these results show that activation of cellular, humoral, and innate compartments prior to immunization is associated with lower NAbs after YF-17D vaccination. These data suggest that activation of the immune microenvironment impedes the adaptive response to YF-17D vaccination.

Decreased persistence of cellular and bumoral immune responses to $Y F-17 D$ in Entebbe vaccines. As we showed a decreased magnitude of cellular and humoral immune responses to YF-17D in Entebbe vaccines, we investigated whether the persistence of YF-17D memory response was also impaired in this cohort. To determine the persistence of YF-17D-specific CD8 $\mathrm{T}$ cell responses after vaccination, we analyzed clonal $\mathrm{V} \beta$ repertoire changes of the $\mathrm{A} 2 / \mathrm{NS} 4 \mathrm{~B}$-specific CD8 $\mathrm{T}$ cells at 2 different time points in the 2 cohorts (Supplemental Table 6). The A2/NS4B-specific CD8 T cell responses were composed of 3 to 7 different $V \beta$ s that were either present at the 2 time points studied or present at a single time point. The number of different A2/NS4B-specific V $\beta$ s was similar for both cohorts, with an average of $4.5 \mathrm{~V} \beta \mathrm{s}$ in the Lausanne cohort and 4.6 in the Entebbe cohort. The A2/NS4B-specific V $\beta$ repertoire in the Lausanne cohort was composed mainly by persisting V $\beta$ s present at the 2 time points, with an average of 2.8 persisting and 1.6 nonpersisting V $\beta$ s. However, in the Entebbe cohort, the repertoire was dominated by nonpersisting $V \beta s$, with an average of 1.5 persisting and 3.1 nonpersisting V $\beta$ s. Overall, the frequency of persisting A2/ NS4B-specific V $\beta$ s present at the 2 time points was significantly lower in YF-17D-naive vaccinated subjects from Entebbe when compared with that in the Lausanne cohort (means: 64 in the Lausanne cohort and 32 in the Entebbe cohort; $P<0.0001$ ) (Figure $8 \mathrm{~A})$. Interestingly, in the Entebbe cohort, the frequencies of persisting A2/NS4B-specific V $\beta$ s at each time point were significantly higher in previously vaccinated volunteers who received a second (boost) vaccine in this study when compared with those in naive vaccinees (mean: 73 in the Entebbe cohort boosted; $P=0.002$ ) (Figure 8A). Of note, YF-17D-specific CD8 T cell responses were boosted by revaccination in the 2 HLA-A*0201-boosted subjects in the Entebbe cohort (Supplemental Figure 9A). We could not assess the second vaccine response in the Lausanne cohort, as the boosted subjects were not HLA-A*0201 in this cohort.

The preimmunized group in the 2 cohorts provided an opportunity to analyze the persistence of NAbs generated from the first YF-17D vaccination. In the Lausanne cohort, high levels of NAbs against YF-17D were detected already at baseline (day 3 and 7) in subjects that had received the YF-17D vaccine 10 years prior to boosting (Figure 8B), and levels remained high after boosting. Of note, 1 subject was not measured for NAb titers at day 7 , and 2 subjects were lost in the follow-up at 1 year. In contrast, YF-17D NAb levels in preimmunized subjects from Entebbe were significantly lower at baseline (day 0 and 7) compared with those at day 

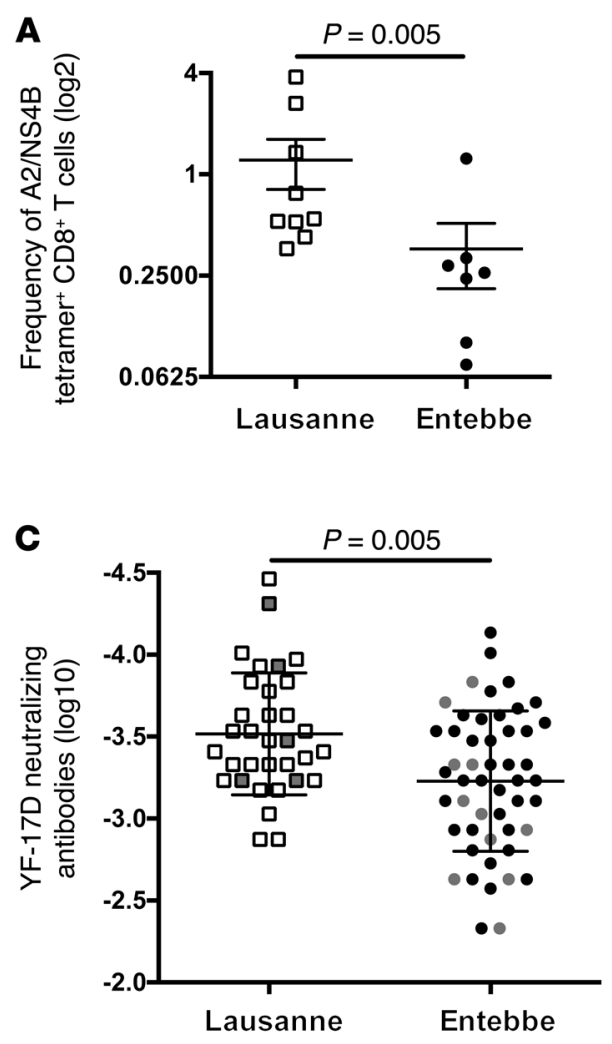
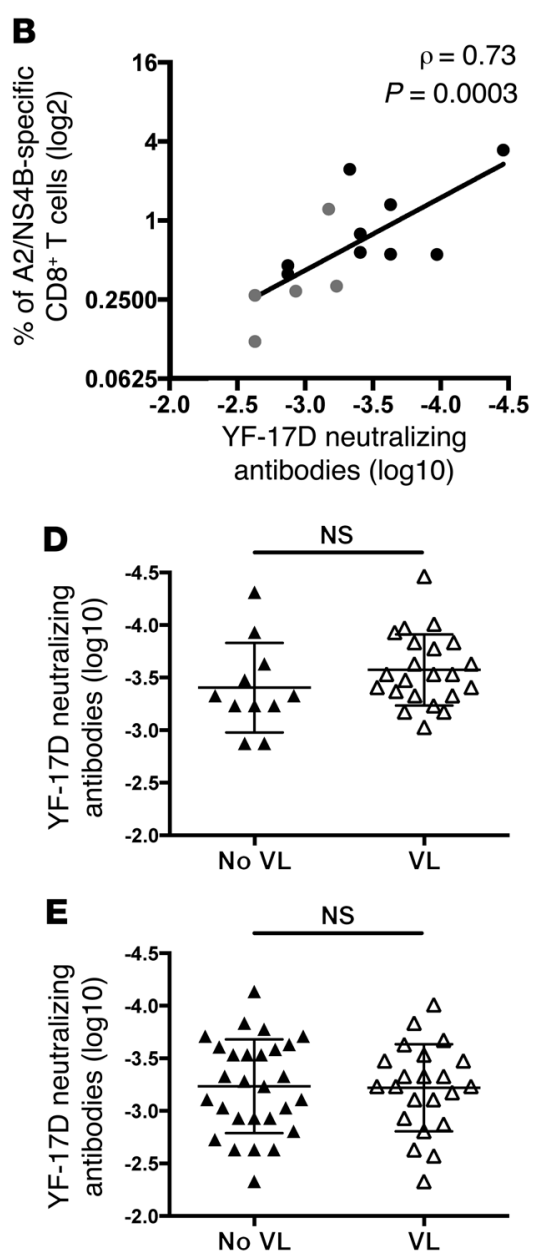

\section{Figure 6}

Differences in A2/NS4B-specific CD8 T cells and NAb titers after YF-17D vaccination between Lausanne and Entebbe cohorts. (A) Frequency of A2/NS4B-specific CD8 T cells in HLA-A*0201 vaccinees from Lausanne (white squares) and Entebbe (black dots) after vaccination. (B) Correlation between YF-17D NAb titers and frequency of A2/NS4B-specific CD8 $T$ cells in both cohorts after vaccination. Gray dots depict samples from the Entebbe cohort. (C) YF-17D NAb titers in vaccinees from Lausanne (white squares) and Entebbe (black dots) after vaccination. Boosted individuals in both cohorts are depicted in gray. (D and E) YF-17D NAb titers in subjects with (white triangles) or without (black triangles) detectable viremia after vaccination (D) in the Lausanne cohort and $(E)$ in the Entebbe cohort.
84 after boosting $(P=0.0006)$ and with the Lausanne cohort at baseline (day 3) $(P=0.0005)$ (Figure $8 \mathrm{~B})$. We observed a significant inverse correlation between the NAb levels prior to vaccination and the increase in YF-17D NAb titers after boosting $(P=$ $0.0005)$, suggesting a negative association between preexisting $\mathrm{NAbs}$ and the boosting of $\mathrm{B}$ cell responses (Figure $8 \mathrm{C}$ ). The date of the first YF-17D vaccination was collected for 10 of the 11 boosted vaccinees from Entebbe. Vaccinees who received the first vaccination less than 7 years prior to the boosting dose showed detectable NAb titers against YF-17D at baseline (Figure 8D). In the Entebbe cohort, YF-17D-neutralizing activity was boosted by revaccination in both subjects with undetectable and detectable NAb titers at baseline (Supplemental Figure 9, B and C). Importantly, 3 of 11 preimmunized subjects in the Entebbe cohort showed detectable viral replication after boosting, whereas YF-17D viral load was not detected in any boosted vaccinees from Lausanne, showing that the protective effect of NAbs persisted for 10 years in the Lausanne cohort (Figure 8E). Of note, the 3 boosted volunteers showing viral replication had no detectable NAbs at baseline, confirming the loss of NAbs after 7 years in the Entebbe cohort. Altogether, these results show a lower persistent cellular and humoral memory response in Entebbe vaccinees when compared with Lausanne vaccinees after YF-17D vaccination. These data suggest that YF-17D immune responses may need to be boosted in Entebbe.

\section{Discussion}

We demonstrated in this study that the immune microenvironment affects the quantitative and qualitative response to YF-17D. The Entebbe cohort showed a reduced magnitude of cellular and humoral responses to the vaccine, as NAb titers and YF-17D-specific CD8 T cells induced 3 months after vaccination were significantly lower than those induced in Lausanne vaccinees after 1 year. YF-17D induced high levels of NAbs in most individuals in Lausanne, whereas its efficacy was more variable in individuals in Entebbe, with a broader range of NAb titers (2 logs in Entebbe and $1 \log$ in Lausanne). We also observed a significantly lower viremia in vaccinees from Entebbe when compared with the Lausanne cohort but did not find an association between the level of viremia and the NAb titers. These data suggest that viral antigen load is not the main determinant for the magnitude of vaccine response. As we showed that the sera of Entebbe volunteers at baseline did not have any neutralizing activity against YF-17D or DENV-2, we could exclude a preexisting immunity of cross-reactive antibodies as the cause of lower vaccine response in Entebbe. We found in this study that neither gender nor viremia affected the YF-17D response. We cannot rule out the impact of polymorphisms in genes involved in cytokines or HLA molecules in the vaccine response (36-39). Studies with greater numbers of volunteers would be required to address their effect on the YF-17D response. Nonetheless, our observations converged to a baseline activated 

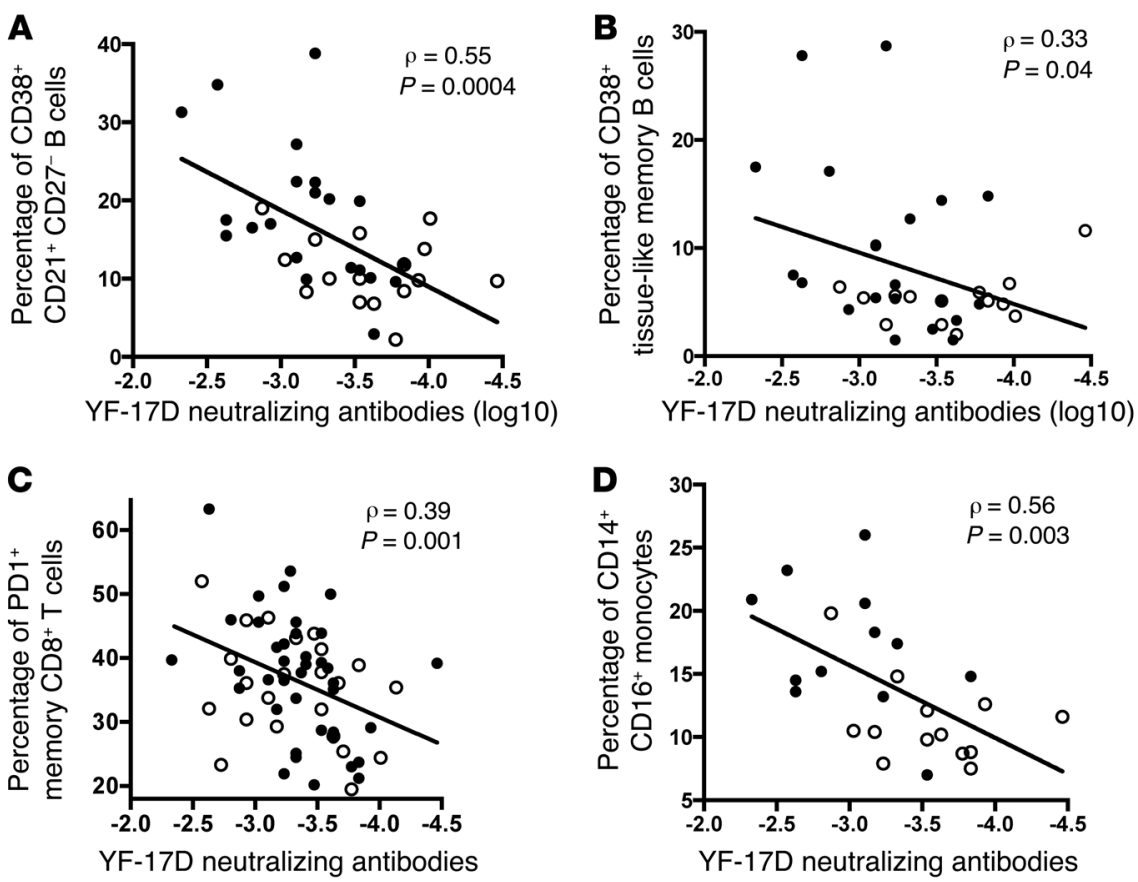

$(\log 10)$

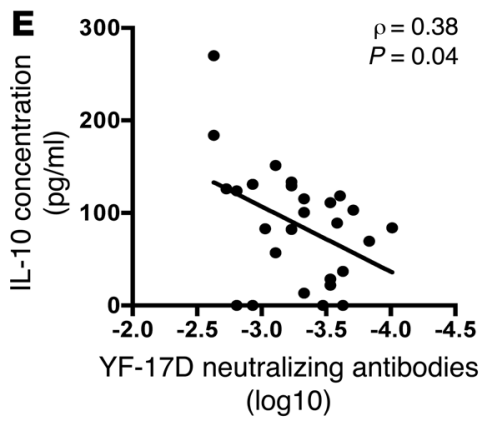

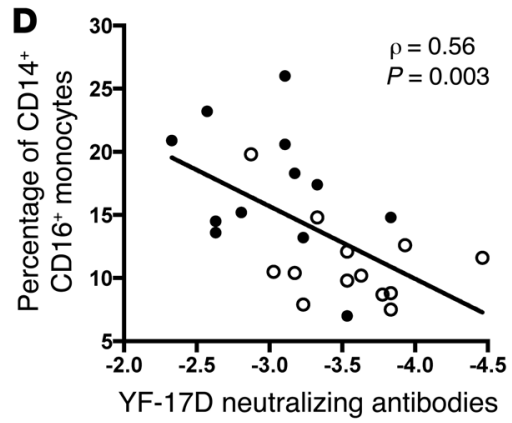

$(\log 10)$

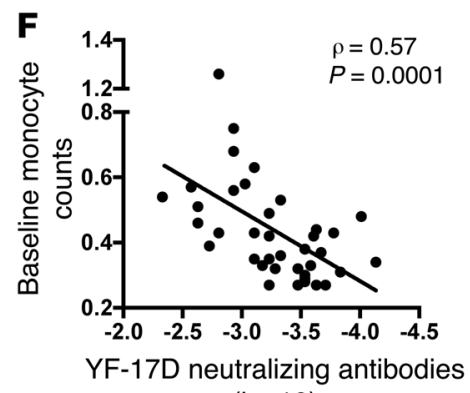

$(\log 10)$
Figure 7

Negative associations between baseline immune activation and YF-17D vaccine response. (A) Negative correlation between the frequency of CD38+CD21+CD27- B cells at baseline and the YF-17D NAb titers in both cohorts. (B) Negative correlation between the frequency of tissue-like memory $B$ cells at baseline and the YF-17D NAb titers in both cohorts. (C) Negative correlation between the expression level of PD-1 on memory CD8 T cells at baseline and the YF-17D NAb titers in both cohorts. (D) Negative correlation between the frequency of CD14+ CD16 ${ }^{+}$monocytes at baseline and the YF-17D NAb titers in both cohorts. Black dots represent data points from the Entebbe cohort, and white dots represent data points from the Lausanne cohort. (E) Negative correlation between the IL-10 secretion in the supernatant of PBMCs at baseline stimulated for 24 hours with YF-17D and the NAb titers in the Entebbe cohort. (F) Negative correlation between the absolute monocyte count at baseline and the YF-17D NAb titers in the Entebbe cohort. immune microenvironment that could limit vaccine replication as well as the magnitude of YF-17D response in the Entebbe cohort. Indeed, we found striking differences in both innate and adaptive compartments between the 2 cohorts. The innate compartment of Entebbe volunteers prior to vaccination displayed significantly higher frequencies of proinflammatory monocytes, exhausted NK cells, and activated NK cells secreting effector molecules. The higher activation of NK cells has been proposed to have a negative impact on the development of $\mathrm{T}$ cell immunity $(40,41)$. In our study, we did not observe correlations between activated or exhausted NK cell subsets at baseline and YF-17D-specific B cell response. However, we observed an association between the frequency of activated NK cells in the Entebbe cohort 7 days after vaccination and the NAb levels (data not shown), suggesting that NK cells might influence the adaptive response to YF-17D after vaccination. The $\mathrm{CD} 14^{+} \mathrm{CD} 16^{+}$monocytes have been shown to be increased dramatically in patients with severe infection $(42,43)$ and are a marker of ongoing activation of the immune system. The adaptive compartment of Entebbe vaccinees displayed more differentiated T and B cells as well as higher frequencies of activated $\mathrm{T}$ and $\mathrm{B}$ cells. Interestingly, we observed increased levels of tissuelike memory B cells in the Entebbe cohort. This B cell subset has been shown to be higher in diseases associated with proinflamma- tory and chronic immune activation, such as HIV infection (31). We also observed an increased frequency of plasmablasts in the Entebbe cohort compared with that in the Lausanne cohort, suggesting an increased proinflammatory environment or a heightened antigen triggering.

Previous reports have demonstrated the role of early innate responses after immunization on the vaccine response outcome for YF-17D $(8,9)$ and also for the Merck Ad5/HIV vaccine (44). However, we demonstrate in this study the role of baseline immune activation prior to immunization in the subsequent vaccine response. The results presented here show a profound activation of innate and adaptive immune cells prior to vaccination that negatively affects the NAb response to YF-17D in the Entebbe cohort. Indeed, we showed that $\mathrm{CD} 38^{+} \mathrm{CD} 21^{+} \mathrm{CD} 27^{-} \mathrm{B}$ cells, tissue-like memory B cells, and PD-1 expression on memory CD8 T cells correlate negatively with NAb titers. CD $38^{+} \mathrm{CD} 21^{+} \mathrm{CD} 27^{-} \mathrm{B}$ cells lacking CD10 as well as PD-1 expression on CD8 T cells have been shown to be increased in HIV infection, in which high immune activation is maintained (45). Furthermore, the proinflammatory monocytes $\mathrm{CD} 14^{+} \mathrm{CD} 16^{+}$and IL-10 levels also strongly negatively correlated with NAb titers. Of note, the potential negative role of monocytes on vaccine response has been suggested in response to hepatitis B virus vaccination (46). We found here that the baseline 

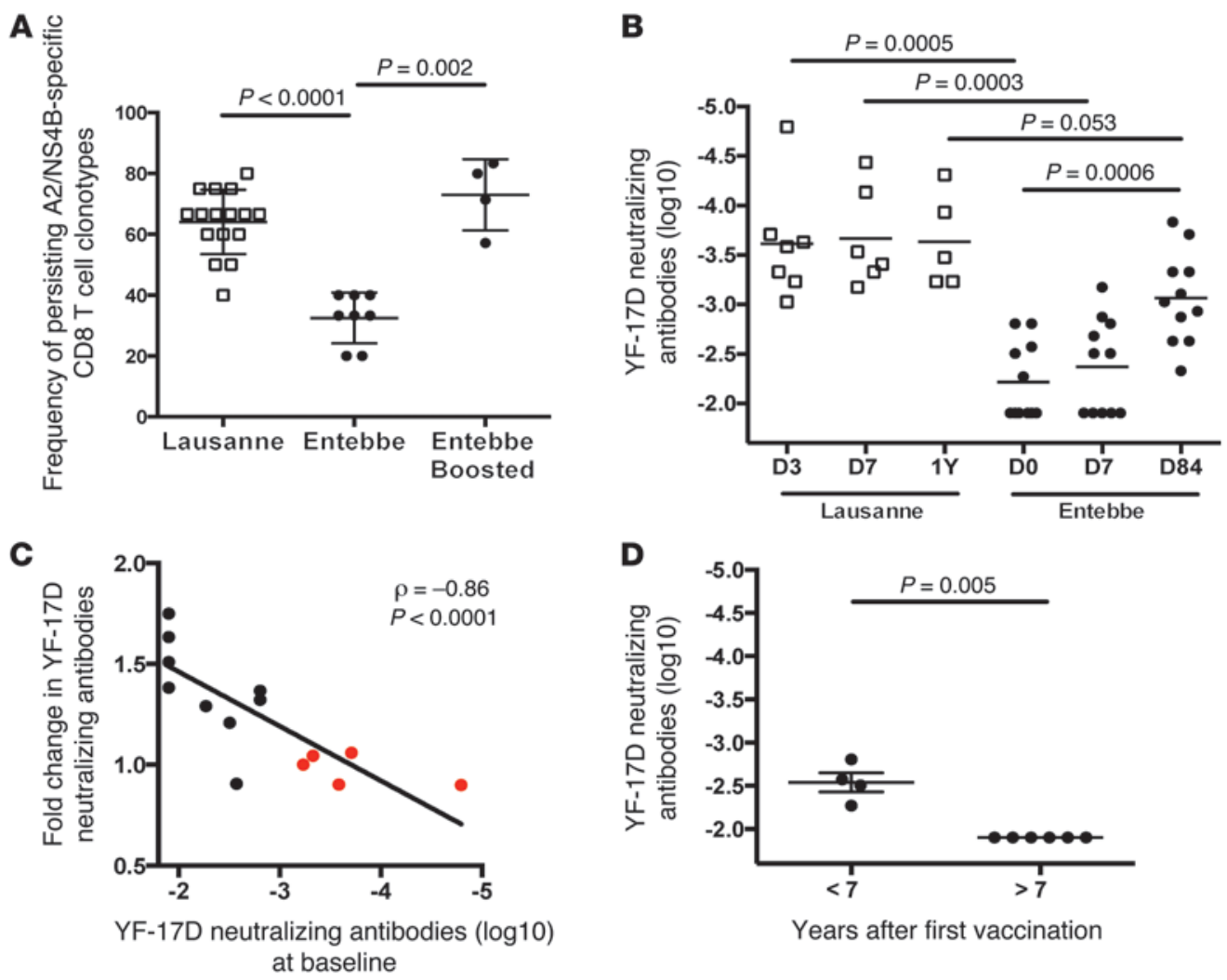

Years after first vaccination

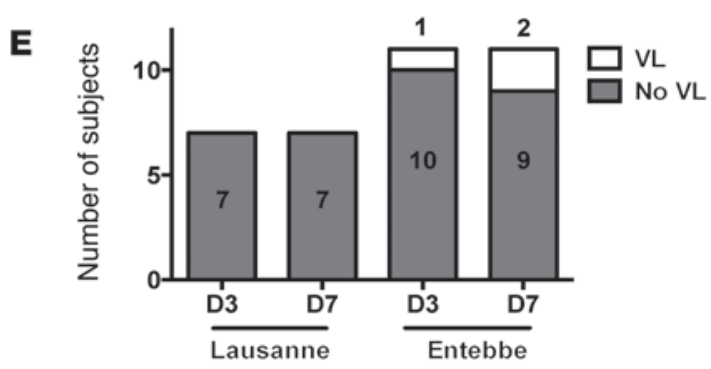

\section{Figure 8}

Quality of the memory T and B cell responses after YF-17D vaccination in the Lausanne and Entebbe cohorts. (A) Frequency of persisting A2/ NS4B-specific CD8 T cell clonotypes at 2 time points after first vaccination in the Lausanne cohort and after first and boosted vaccination in the Entebbe cohort. (B) YF-17D NAb titers in the sera of boosted vaccinees in Lausanne and Entebbe cohorts longitudinally at baseline (day 3 or 0 ), day 7, and 1 year or day 84 after vaccination. Empty squares represent data points from the Lausanne cohort, and black dots represent data points from the Entebbe cohort. (C) Negative association between the NAb levels detected at baseline in Lausanne and Entebbe cohorts and the magnitude of NAb boosting after YF-17D revaccination. Red dots depict Lausanne subjects. (D) In the boosted subjects from Entebbe, NAb titers against YF-17D could not be detected at baseline when the first vaccination was 7 years or more in the past. (E) Number of boosted vaccinees with or without detectable viremia in the plasma after YF-17D vaccination in Lausanne and Entebbe cohorts. Numbers of subjects in each group are indicated within the bar graphs.

monocyte count from the blood in the Entebbe cohort was significantly associated with lower NAbs after YF-17D vaccination. This parameter could be further evaluated as a predictor of vaccine outcome, as it is easily quantifiable.

We demonstrated in this study that the activated immune microenvironment in the Entebbe cohort was associated with lower magnitudes of cellular and humoral responses to YF-17D and, even more importantly, with the reduced persistence of these YF-17D memory responses. In the preimmunized subjects from the Lausanne cohort, the NAb levels detected 10 years after vaccination were similar to the levels induced 1 year after vaccination of the naive population, and no change in NAb titers was observed in these revaccinated individuals, suggesting that YF-17D induces high levels of persisting NAb and memory B cells in this group, supporting previous reports $(10,47)$. Strikingly, in the preimmunized individuals from the Entebbe cohort, low NAb titers were detectable 6 years after vaccination but not after longer periods of time, suggesting a decay in NAb titers and a loss of memory $\mathrm{B}$ cell response over time. This may be related to the lower frequency of $\mathrm{IgM}^{+}$memory B cells observed in the Entebbe cohort. In the Entebbe cohort, the vaccine boost resulted in an increase in NAb titers with a trend toward lower NAb levels $(P=0.08)$ 
after a second vaccination, suggesting that the remaining immunity against YF-17D might dampen vaccine efficacy. We further observed a negative association between the preexisting antibodies against YF-17D and the magnitude of $\mathrm{B}$ cell boost after revaccination. Nonetheless, our observations suggest that different revaccination schedules should be implemented in different groups, depending on the persistence of their NAb titers. The correlates of protection of the YF-17D vaccine are difficult to establish in the absence of efficacy studies in humans. Only 5 cases of YFV infection have been reported in YF-17D vaccine recipients (4). However, yellow fever challenge in macaques vaccinated with YF-17D established that the level of protection required is log10(neutralization index) $>0.7$ (48). Even though we cannot link the decrease of NAb titers over time in the Entebbe cohort to a loss of protection against YFV, we observed a detectable viremia in 3 of the 11 boosted volunteers, demonstrating that the previous vaccination did not prevent replication of the attenuated vaccine and might not prevent the replication of the highly replicative wild-type YFV.

The increased activation of immune microenvironment in the Entebbe cohort, which quantitatively and qualitatively affects the cellular and humoral YF-17D responses, might also reduce the efficacy of other vaccines. It would be important to assess whether this impaired adaptive immune response to YF-17D in the Entebbe cohort is valid for other live-attenuated vaccines and whether it is also applicable to killed vaccines. This baseline immune activation status in Entebbe could be due to several causes that would be difficult to tease apart in human populations. One main inducer of constant immune activation is the more frequent exposure to infectious diseases in Entebbe, which lies on the shore of Lake Victoria, when compared with the Lausanne population. Chronic or acute inflammatory conditions, including pathogenic viral, bacterial, fungal, or parasitic infections, might play a role in this immune activation $(49,50)$. Of note, we did not observe significant differences in YF-17D NAb titers between individuals who reported a coinfection as an adverse event after vaccination and those that did not. Other factors, such as diet and gut microbiota, could also play a role. Clinical trials controlling for one or more of these parameters might help decipher the main causes for an activated immune microenvironment in the Entebbe population. Here, we analyzed one population in Uganda, but other studies might be needed to assess whether different levels of immune activation are found in different African populations. Specific immune modulatory strategies might be useful to potentiate the vaccine responses and memory persistence in these populations with an apparent higher baseline immune activation status.

\section{Methods}

Human subjects and YF-17D vaccine. This study enrolled subjects vaccinated with YF-17D at 2 different sites: the UVRI-IAVI in Entebbe and the Travellers clinic in Lausanne. The enrolled volunteers in the 2 studied cohorts were vaccinated with the YF-17D vaccine from Sanofi Pasteur. The immunogenicity of 2 different vaccine batches had been assessed in previous reports and showed that 2 vaccine batches provided equivalent protection $(51,52)$. The yellow fever vaccine used (Stamaril) is a live-attenuated preparation of the 17D strain of YFV (CDC, 1974) and is licensed in Uganda by the National Drug Authority. In Uganda, research participants were recruited from within Entebbe and Kampala through information presented to them in community meetings, travel clinics, hospitals, colleges, and other institutions and/or by advertisements to the general pub- lic. Individuals willing to participate were invited for a screening visit at UVRI-IAVI. Volunteers received the vaccine from August to October 2009 prior to the first documented YFV outbreak in Northern Uganda in October 2010 (53). In Lausanne, research participants were recruited from the Travellers clinic. Volunteers received the vaccine either alone or concomitant with other vaccinations. This study was conducted in compliance with International Conference on Harmonization/good clinical practice, GCLP, and applicable regulatory requirements.

YF-17D production for in vitro assays. The YF-17D initial stock was obtained through the NIH Biodefense and Emerging Infections Research Resources Repository, NIAID, NIH: YFV, 17D, NR-115. YF-17D virus stocks were produced by infecting Vero cell monolayers grown in DMEM medium (Sigma-Aldrich), supplemented with 10\% X-Vivo 15 medium (Lonza), with the YF-17D initial stock (NR-155, BEI Resources). Virus-containing supernatants were harvested when severe destruction of the monolayers was observed (6-7 days). They were cleared twice by centrifugation $(750 \mathrm{~g}$, 10 minutes) and filtered through $0.45-\mu \mathrm{m}$ PVDF syringe filters (Millipore). The viral particles were concentrated using Centricon Plus-20 columns (100-kDa cutoff; Millipore) and titrated on Vero cells by lysis plaque assay. YF-17D viral load. YF-17D viral load was measured by ultrasensitive quantitative RT-PCR, as described previously (54), with slight modification. Viral RNA was extracted from $1 \mathrm{ml}$ plasma with the Total Nucleic Acid Large Volume Extraction Kit (Roche Diagnostics) and was eluted in $65 \mu \mathrm{l}$ elution buffer. The assay's detection limit is 37 copies per ml of plasma.

YF-17D NAb titers. Sera from the 2 cohorts were used to measure the NAb titers. Briefly, serum samples were complement inactivated by means of 30 minutes of incubation at $56^{\circ} \mathrm{C}$ and were then serially diluted in triplicates in culture medium in 96-well flat-bottomed plates. One thousand plaque-forming units of YF-17D were added to each well and incubated at $37^{\circ} \mathrm{C}$ for 2 hours, after which 5,000 Vero cells were added. The plates were incubated at $37^{\circ} \mathrm{C}$ for 7 days, and each well was then observed under an inverted microscope and scored for cytopathic effect as positive (destroyed monolayer) or negative (intact monolayer). The highest dilution resulting in an intact monolayer was used as the antibody titer. YF-17D NAb titers are expressed in $\log 10$ (highest dilution).

Analysis of A2/NS4B-specific CD8 T cells. Subjects from Lausanne and Entebbe cohorts were HLA typed using a 4-digit resolution in Centre Hospitalier Universitaire Vaudois and UVRI-IAVI, respectively. Monomers of HLA-A*0201 were refolded with the YFV NS4B peptide (LLWNGP$\mathrm{MAV}$ ) and tetramerized freshly for each experiment with extravidin-PE (Sigma-Aldrich). Total CD8 T cells from HLA-A*0201-positive subjects in both cohorts were negatively selected by magnetic bead enrichment (Stemcell) and expanded for 2 weeks in the presence of PHA, feeders, and IL-2. Frequency of A2/NS4B-specific CD8 T cells was determined by staining for 15 minutes at $37^{\circ} \mathrm{C}$ with tetramers. $\mathrm{V} \beta$ usage was determined by staining the A2/NS4B-specific CD8 T cells with the BetaMark TCR V Kit (Beckman Coulter) and analyzed by flow cytometry. $\mathrm{V} \beta$ usage was considered positive with a frequency of $>3 \%$.

$D E N V-2$ neutralizing assay. The Macaca mulatta kidney epithelial cell line LLC-MK 2 (ATCC) was grown in DMEM 10\% FBS. DENV-2 strain NG-2 was obtained from R. Tesh at the University of Texas at Galveston, Galveston, Texas, USA. Virus was propagated in LLC-MK $\mathrm{M}_{2}$ cells as previously described (55). For the focus-forming-unit neutralization assay, LLC-MK $\mathrm{M}_{2}$ target cells were seeded at a density of approximately 500,000 cells in each well of a 12-well plate 24 hours prior to DENV inoculation. Approximately 100 focus forming units of DENV were incubated with dilutions of heat inactivated patient serum in serum-free DMEM for 1 hour at $37^{\circ} \mathrm{C}$. DENV/ serum mixtures were allowed to infect confluent target cell monolayers for 1 hour at $37^{\circ} \mathrm{C}$, with rocking every 15 minutes, after which time the inoculum was aspirated and overlaid with fresh MEM/10\% FBS containing 
$1.2 \%$ microcrystalline cellulose (Avicel). Infected cells were then incubated at $37^{\circ} \mathrm{C}$ with $5 \% \mathrm{CO}_{2}$ for 3 days. Infected cultures were fixed with $10 \%$ formalin overnight at $4^{\circ} \mathrm{C}$, permeabilized with $70 \%$ ethanol for 20 minutes, and rinsed with PBS prior to immunostaining. Virus foci were detected using $1 \mathrm{mg} / \mathrm{ml}$ anti-DENV E protein human monoclonal antibody D11C (55) overnight at room temperature, followed by $2 \mathrm{mg} / \mathrm{ml}$ goat anti-human HRP (ThermoScientific) overnight at room temperature, and developed using 3,3'-diaminobenzidine tetrahydrochloride (Sigma-Aldrich). Results are expressed as the average \pm SD from at least 3 replicates each.

Flow cytometry analysis. For the phenotypic analysis of NK cells, thawed PBMCs for each donor and each time point remained unstimulated for phenotyping or were stimulated with PMA/ionomycin for IFN- $\gamma$ staining and were incubated with $1 \mu \mathrm{g} / \mathrm{ml}$ GolgiPlug (BD Biosciences) and $5 \mu \mathrm{g} / \mathrm{ml}$ Brefeldin (BD Biosciences) for 5 hours at $37^{\circ} \mathrm{C}$. Cells were then stained for surface markers at $4^{\circ} \mathrm{C}$ for 20 minutes with the following monoclonal antibodies: NKp46 PE (BD Pharmingen, 9E2/Nkp46), CD56 PECy7 (BD Pharmingen, B159), CD16 V450 (BD Horizon, 3G8), and HLA-DR PerCP Cy5.5 (BD Pharmingen, G46-6 L243). Permeabilization and fixation was performed using the Cytofix/Cytoperm Kit (BD 554722), and intracellular staining was performed with IFN- $\gamma$ Alexa Fluor 700 (BD Pharmingen, B27) monoclonal antibody for 30 minutes at room temperature, while protected from light. For the phenotypic analysis of CD4 and CD8 T cells, thawed PBMCs were stained for surface markers at $4^{\circ} \mathrm{C}$ for 20 minutes with the following monoclonal antibodies: CD8-FITC, HLA-DR-PerCP, CCR7-PE, PD1-PECy7, CD38-APC, CD3-Alexa Fluor 700, CD45RA-APCH7, CD4Qdot-655, and CD27-Qdot605 (BD Biosciences, Invitrogen). For the phenotypic analysis of CD4 Tregs cells and B cell subsets, thawed PBMCs were stained for surface markers at $4{ }^{\circ} \mathrm{C}$ for 20 minutes with the following monoclonal antibodies: CD3-A700, CD4-APC Cy7, and CD45RAECD (Beckman Coulter); CD25-PE Cy5, CD38, PerCP, ICOS-PE, PD-1PE Cy7, CD127-Brilliant Violet 421, and FoxP3-FITC (eBioscience); and CD19-A700, CD27-PE, CD21-Pacific Blue, IgM-PE Cy5, and IgG-PE Cy7 (BD Biosciences). For monocyte staining, FcR blocking was first performed using human TruStain FcX (BioLegend), followed by surface staining with the following monoclonal antibodies at $4{ }^{\circ} \mathrm{C}$ for 20 minutes: $\mathrm{CD} 14-\mathrm{PerCP}$ (R\&D Systems), CD16-A700 (BD Biosciences), CD3-Pacific Blue, HLADR APC Cy7, CD19-PE Cy7, PD-L1-APC and PD-L2-PE. All antibodies were from BioLegend unless otherwise noted. Live/Dead Fixable Aqua (Invitrogen) was used to exclude dead cells from the analysis. Data were collected using an LSRII flow cytometer (BD Biosciences) and analyzed with FlowJo software (version 9.6.2, TreeStar Inc.) and DIVA software (version 8.0, BD Biosciences).

Cytokine secretion analysis. Cytokine secretion of PBMCs, incubated or not with YF-17D at MOI 5 for 24 hours, was detected in the supernatant by the CBA Human Th1/Th2 Cytokine Kit, according to the manufacturer's instructions. Data were collected using a FACSArray (BD Biosciences) and analyzed with BD FCAP array software.

Statistics. In all dot plots, horizontal bars represent the mean and the error bars represent the SEM. A nonparametric Mann-Whitney test was used to compare groups, and differences were considered significant at $P<0.05$.
A $\chi^{2}$ test was used to compare distributions of groups, and differences were considered significant at $P<0.05$. To find association between the baseline innate and adaptive parameters and the levels of YF-17D response, we performed a systematic correlation test between the NAb titers and every parameter analyzed in the study and available clinical data. Correlations analysis was performed using a nonparametric Spearman test, and correlations were indicated with the spearman $\rho$ value and the $P$ value. All significant correlations are described in the manuscript.

Study approval. The study was approved by the UVRI Science and Ethics Committee and the Uganda National Council for Science and Technology, Kampala, Uganda. The ethics committee is registered with the US Office of Human Research Protection. The study was also approved by the Ethics Committee of Clinical Research from Lausanne University and Centre Hospitalier Universitaire Vaudois, Lausanne, Switzerland. Written informed consent was obtained from each participant prior to conducting any study procedures.

\section{Acknowledgments}

The following reagent was obtained through the NIH Biodefense and Emerging Infections Research Resources Repository, NIAID, NIH: Yellow Fever Virus (YFV), 17D, NR-115. We would like to thank Denis Gaucher for the YF-17D production and Santosh Menon for the A2/NS4B monomer production. We would like to thank Bob O'Neill, former director of the Canadian HIV Trials Network, International Program, for support in the coordination of the study. We would like to acknowledge the travel medicine consultation from the Polyclinique Médicale Universitaire de Lausanne for their collaboration in volunteer recruitment. This work was part of the Canada-Africa Prevention Trials Network activities funded by Canada's Global Health Research Initiative. This work was also supported by the CIHR Canadian HIV Trials Network; funds from the Office of Tourism, Trade and Economic Development of Florida; the Defense Threat Reduction Agency under award number HDTRA1-10-1-0009; the NIH National Institute of Allergy and Infectious Diseases under award number R01AI099210; the Emory Center for AIDS Research under award number P30 AI050409; and the Bill \& Melinda Gates Foundation grants SEKALY06VIMC0 and GH-HTR-05-02. This study was made possible by the support of the American people through the United States Agency for International Development. The contents of the paper are the responsibility of the authors and the International AIDS Vaccine Initiative and do not necessarily reflect the views of USAID, the NIH, or the United States government.

Received for publication January 28, 2014, and accepted in revised form April 24, 2014.

Address correspondence to: Lydie Trautmann, VGTI Florida, 9801 SW Discovery Way, Port Saint Lucie, Florida 34987, USA. Phone: 772.345.5671; Fax: 772.345.0625; E-mail: 1trautmann@vgtifl.org.
1. Poland JD, Calisher CH, Monath TP, Downs WG, Murphy K. Persistence of neutralizing antibody 30-35 years after immunization with $17 \mathrm{D}$ yellow fever vaccine. Bull World Health Organ. 1981;59(6):895-900.

2. Monath TP, Barrett AD. Pathogenesis and pathophysiology of yellow fever. Adv Virus Res. 2003;60:343-395.

3. Niedrig M, Lademann M, Emmerich P, Lafrenz M. Assessment of IgG antibodies against yellow fever virus after vaccination with $17 \mathrm{D}$ by different assays: neutralization test, haemagglutination inhibition test, immunofluorescence assay and ELISA. Trop Med Int Health. 1999;4(12):867-871.
4. Monath TP, Cetron MS, Teuwen DE. Yellow fever vaccine. In: Plotkin SA, Orenstein WA, Offit PA, eds. Vaccines. 5th ed. Philadelphia, Pennsylvania, USA: Saunders Elsevier; 2008:959-1055.

5. Staples JE, Gershman M, Fischer M. Yellow fever vaccine: recommendations of the Advisory Committee on Immunization Practices (ACIP). MMWR Recomm Rep. 2010;59(RR-7):1-32.

6. Gotuzzo E, Yactayo S, Cordova E. Efficacy and duration of immunity after yellow fever vaccination: systematic review on the need for a booster every 10 years. Am J Trop Med Hyg. 2013;89(3):434-444.
7. World Health Organization. Yellow fever fact sheet $\mathrm{N}^{\circ} 100$. WHO Web site. http://www.who.int/ mediacentre/factsheets/fs100/en/. Updated March 2014. Accessed May 13, 2014.

8. Querec TD, et al. Systems biology approach predicts immunogenicity of the yellow fever vaccine in humans. Nat Immunol. 2008;10(1):116-125.

9. Gaucher D, et al. Yellow fever vaccine induces integrated multilineage and polyfunctional immune responses. J Exp Med. 2008;205(13):3119-3131.

10. Reinhardt B, Jaspert R, Niedrig M, Kostner C, L'age-Stehr J. Development of viremia and humoral 
and cellular parameters of immune activation after vaccination with yellow fever virus strain 17D: a model of human flavivirus infection. J Med Virol. 1998;56(2):159-167.

11. Silva ML, et al. Characterization of main cytokine sources from the innate and adaptive immune responses following primary 17DD yellow fever vaccination in adults. Vaccine. 2011;29(3):583-592.

12. Barba-Spaeth G. Live attenuated yellow fever $17 \mathrm{D}$ infects human DCs and allows for presentation of endogenous and recombinant T cell epitopes. J Exp Med. 2005;202(9):1179-1184

13. Martins MA, et al. Activation/modulation of adaptive immunity emerges simultaneously after 17DD yellow fever first-time vaccination: is this the key to prevent severe adverse reactions following immunization? Clin Exp Immunol. 2007;148(1):90-100.

14. Mandl JN, et al. Distinctive TLR7 signaling, type I IFN production, and attenuated innate and adaptive immune responses to yellow fever virus in a primate reservoir host. J Immunol. 2011;186(11):6406-6416.

15. Co MDT, Terajima M, Cruz J, Ennis FA, Rothman AL. Human cytotoxic T lymphocyte responses to live attenuated $17 \mathrm{D}$ yellow fever vaccine: identification of HLA-B35-restricted CTL epitopes on nonstructural proteins NS1, NS2b, NS3, and the structural protein E. Virology. 2002;293(1):151-163.

16. Santos APD, Bertho AL, Dias DC, Santos JR, Marcovistz R. Lymphocyte subset analyses in healthy adults vaccinated with yellow fever $17 \mathrm{DD}$ virus. Mem Inst Oswaldo Cruz. 2005;100(3):331-337.

17. Miller JD, et al. Human effector and memory CD8+ $\mathrm{T}$ cell responses to smallpox and yellow fever vaccines. Immunity. 2008;28(5):710-722.

18. Akondy RS, et al. The yellow fever virus vaccine induces a broad and polyfunctional human memory CD8+ T cell response. JImmunol. 2009;183(12):7919-7930.

19. Rappuoli R, Aderem A. A 2020 vision for vaccines against HIV, tuberculosis and malaria. Nature. 2011;473(7348):463-469.

20. Bonaldo MC, et al. Recombinant yellow fever vaccine virus $17 \mathrm{D}$ expressing simian immunodeficiency virus SIVmac239 gag induces SIV-specific CD8+ T-cell responses in rhesus macaques. J Virol. 2010;84(7):3699-3706

21. Guy B, et al. Preclinical and clinical development of YFV 17D-based chimeric vaccines against dengue, West Nile and Japanese encephalitis viruses. Vaccine. 2010;28(3):632-649

22. Martins MA, et al. Immunogenicity of seven new recombinant yellow fever viruses $17 \mathrm{D}$ expressing fragments of SIVmac239 Gag, Nef, and Vif in Indian rhesus macaques. PLoS One. 2013;8(1):e54434.

23. Monath TP, et al. Comparative safety and immunogenicity of two yellow fever 17D vaccines (ARILVAX and YF-VAX) in a phase III multicenter, double-blind clinical trial. Am J Trop Med Hyg. 2002;66(5):533-541.

24. Monath TP, et al. Yellow fever 17D vaccine safety and immunogenicity in the elderly. Hum Vaccin.
2005;1(5):207-214.

25. Roukens AH, et al. Elderly subjects have a delayed antibody response and prolonged viraemia following yellow fever vaccination: a prospective controlled cohort study. PLoS One. 2011;6(12):e27753.

26. Black GF, et al. BCG-induced increase in interferon-gamma response to mycobacterial antigens and efficacy of BCG vaccination in Malawi and the UK: two randomised controlled studies. Lancet. 2002;359(9315):1393-1401.

27. Lalor MK, et al. BCG vaccination induces different cytokine profiles following infant BCG vaccination in the UK and Malawi. $J$ Infect Dis. 2011;204(7):1075-1085.

28. Gilmour J, et al. Immunogenicity of homologous and heterologous regimens of Ad26-Enva.01 and Ad35-Enva HIV vaccines in HIV-uninfected volunteers in the U.S. and Africa. AIDS Res Hum Retroviruses. 2013;29(11):A69.

29. Bhatt $S$, et al. The global distribution and burden of dengue. Nature. 2013;496(7446):504-507.

30. Ziegler-Heitbrock HWL. Heterogeneity of human blood monocytes: the CD14+CD16+ subpopulation. Immunol Today. 1996;17(9):424-428.

31. Moir S, et al. Normalization of B cell counts and subpopulations after antiretroviral therapy in chronic HIV disease. J Infect Dis. 2008;197(4):572-579.

32. Moir S, et al. B cells in early and chronic HIV infection: evidence for preservation of immune function associated with early initiation of antiretroviral therapy. Blood. 2010;116(25):5571-5579.

33. Reynaud C-A, et al. IgM memory B cells: a mouse/human paradox. Cell Mol Life Sci. 2012; 69(10):1625-1634

34. Yates JL, Racine R, McBride KM, Winslow GM. T cell-dependent IgM memory B cells generated during bacterial infection are required for $\mathrm{IgG}$ responses to antigen challenge. JImmunol. 2013;191(3):1240-1249.

35. de Melo AB, et al. T-cell memory responses elicited by yellow fever vaccine are targeted to overlapping epitopes containing multiple HLA-I and -II binding motifs. PLoS Negl Trop Dis. 2013;7(1):e1938.

36. Ovsyannikova IG, Dhiman N, Jacobson RM, Poland GA. Human leukocyte antigen polymorphisms: variable humoral immune responses to viral vaccines. Expert Rev Vaccines. 2006;5(1):33-43.

37. Yucesoy $\mathrm{B}$, et al. Influence of cytokine gene variations on immunization to childhood vaccines. $\mathrm{Vac}$ cine. 2009;27(50):6991-6997.

38. Haralambieva IH, et al. The genetic basis for interindividual immune response variation to measles vaccine: new understanding and new vaccine approaches. Expert Rev Vaccines. 2013;12(1):57-70.

39. Yucesoy B, et al. Genetic variants within the MHC region are associated with immune responsiveness to childhood vaccinations. Vaccine. 2013; 31(46):5381-5391.

40. Lang PA, et al. Natural killer cell activation enhances immune pathology and promotes chronic infection by limiting CD8+ T-cell immunity. Proc Natl Acad Sci US A. 2012;109(4):1210-1215.

41. Waggoner SN, Cornberg M, Selin LK, Welsh RM. Natural killer cells act as rheostats modulating antiviral T cells. Nature. 2012;481(7381):394-398.

42. Fingerle G, et al. The novel subset of CD14+/CD16+ blood monocytes is expanded in sepsis patients. Blood. 1993;82(10):3170-3176.

43. Nockher WA, Scherberich JE. Expanded CD14+ CD16+ monocyte subpopulation in patients with acute and chronic infections undergoing hemodialysis. Infect Immun. 1998;66(6):2782-2790.

44. Zak DE, et al. Merck Ad5/HIV induces broad innate immune activation that predicts CD8+ T-cell responses but is attenuated by preexisting Ad5 immunity. Proc Natl Acad Sci U S A. 2012; 109(50):E3503-E3512.

45. Moir S, Fauci AS. B cells in HIV infection and disease. Nat Rev Immunol. 2009;9(4):235-245.

46. Anthony DD, et al. Lower peripheral blood CD14+ monocyte frequency and higher CD34+ progenitor cell frequency are associated with HBV vaccine induced response in HIV infected individuals. Vaccine. 2011;29(19):3558-3563.

47. Wisseman CL, Sweet BH. Immunological studies with group B arthropod-borne viruses. III. Response of human subjects to revaccination with 17D strain yellow fever vaccine. Am J Trop Med Hyg. 1962;11:570-575.

48. Mason RA, Tauraso NM, Spertzel RO, Ginn RK. Yellow fever vaccine: direct challenge of monkeys given graded doses of $17 \mathrm{D}$ vaccine. Appl Microbiol. 1973;25(4):539-544.

49. Ben-Smith A, et al. Differences between naive and memory $\mathrm{T}$ cell phenotype in Malawian and UK adolescents: a role for Cytomegalovirus? BMC Infectious Diseases. 2008;8(1):139.

50. Stelekati E, Wherry EJ. Chronic Bystander Infections and Immunity to Unrelated Antigens. Cell Host and Microbe. 2012;12(4):458-469.

51. Lang J, et al. Comparison of the immunogenicity and safety of two $17 \mathrm{D}$ yellow fever vaccines. $A m J$ Trop Med Hyg. 1999;60(6):1045-1050.

52. Barban V, et al. High stability of yellow fever 17D-204 vaccine: a 12-year retrospective analysis of large-scale production. Vaccine. 2007;25(15):2941-2950.

53. Wamala JF, et al. Epidemiological and laboratory characterization of a yellow fever outbreak in northern Uganda, October 2010-January 2011. Int J Infect Dis. 2012;16(7):e536-e542.

54. Edupuganti S, et al. A randomized, double-blind, controlled trial of the $17 \mathrm{D}$ yellow fever virus vaccine given in combination with immune globulin or placebo: comparative viremia and immunogenicity. Am J Trop Med Hyg. 2013;88(1):172-177.

55. Costin JM, et al. Mechanistic study of broadly neutralizing human monoclonal antibodies against dengue virus that target the fusion loop. J Virol. 2013;87(1):52-66 